\title{
Early Outcomes of Transoral Laser Microsurgery for T1-T2 Oropharyngeal Cancer
}

\author{
Tran Phan Chung Thuy and Nguyen Thanh Tuan* \\ School of Medicine, Vietnam National University - Ho Chi Minh City, Linh Trung Ward, Thu Duc District, \\ Ho Chi Minh City - 700000, Vietnam; nttuan@medvnu.edu.vn
}

\begin{abstract}
The optimal treatment strategy for oropharyngeal carcinoma is highly debated. However, growing evidence supports the use of minimally invasive techniques, such as Transoral Laser Microsurgery (TLM), as a first-line treatment modality for these carcinomas. The purpose of our study was to assess the efficacy and safety of TLM for the treatment of early oropharyngeal carcinomas. This is a prospectively observational study of 30 early stages (T1/T2) oropharyngeal cancer patients who underwent transoral laser microsurgery from January 2016 to March 2019. 30 patients of early-staged oropharyngeal carcinoma (19 tongues, 5 soft palates, 4 tonsils and 2 posterior pharyngeal walls) were included in this study. 19 (63.3\%) patients were male and $11(36.7 \%)$ were female. The mean age was 54.8 years ( \pm 10.6 years). $76.7 \%$ tumours are $\mathrm{T} 1$ and $23.3 \% \mathrm{~T} 2$. The bleeding complications occurred in 2 patients. The most frequent complication of TLM was bleeding. $30 \%$ of patients had normal voices and a further $63.3 \%$ had only mild or moderate voice change. At their last follow up, no patients had any difficulty in breathing or swallowing. TLM is a safe, minimally invasive and effective method in the treatment for early oropharyngeal cancer.
\end{abstract}

Keywords: Carbon Dioxide Laser, Oropharyngeal Carcinoma, Transoral Laser Microsurgery

\section{Introduction}

Transoral Laser Microsurgery (TLM) has been accepted as a major treatment modality for early oropharyngeal carcinoma (T1, T2), with well-known advantages over Radiation Therapy (RT) and open surgical procedures ${ }^{1-3}$. Besides these well-established oncological approaches, transoral laser surgery has emerged as a major therapeutic alternative. Such a preference seems quite obvious considering the advantages of low morbidity, good functional results, lower costs, and shorter hospital stay without significant differences in local control or survival compared to other modalities ${ }^{4-6}$. Endoscopic laser surgery for oropharyngeal cancer was first described and used in the United States and accepted widely in clinical practice in Europe. The initial pioneer work by Steiner and subsequent major studies by Motta et al. Rudert and Werner ${ }^{13}$ have demonstrated the potential use of transoral laser surgery (TLS) for early-stage oropharyngeal carcinoma ${ }^{1}$. However, in Vietnam, no detailed information on local and regional control, final organ preservation rates and survival is available to date that might permit a meaningful comparison of TLS to other treatment modalities for early oropharyngeal carcinoma. In this prospective study, we evaluated oncologic outcomes in a consecutive series of early oropharyngeal carcinoma treated with TLS as a singlemodality therapy and analyzed the relationship between these outcomes and other clinicopathologic factors. Main outcome measures were local control with initial therapy.

*Author for correspondence 


\section{Patients and Methods}

We performed a prospective study of the medical records of 30 consecutive patients with previously untreated early stage (T1/T2 N0M0) oropharyngeal carcinoma who underwent TLM at the Ho Chi Minh City ENT Hospital, Vietnam, from January 2016 to March 2018 without any adjuvant treatment. The patients had been diagnosed with Squamous Cell Carcinoma (SCC) by tissue biopsy with direct pharyngoscopy conducted under local anesthesia before the TLM. The surgical exposure of the pharynx via the microscope was evaluated during the procedure. The surgeon made efforts to visualize the lesions in patients with difficult oropharyngeal exposure. Most of the cases were successfully exposed with such efforts. Neck Computed Tomography (CT) scans was performed for patients with involvement of regional extension. Informed consent for the procedure was obtained from patients after explaining the treatment choice of RT and TLM. In patients who had been consented prior to the biopsy procedure, a single-stage TLM was performed with both diagnostic and therapeutic purposes based on an intra-operative frozen section.

\subsection{Surgical Technique}

The surgical technique for the transoral approach to early-stage oropharyngeal carcinoma used throughout this study has previously been described in detail. In brief, The TLM was performed under microscopy using a $\mathrm{CO}_{2}$ laser (Lumenis, Israel) coupled with a micromanipulator (AcuSpot 715) with super pulse delivery in continuous mode ( $4-8 \mathrm{~W}$ ) at a spot size of approximately $0.8 \mathrm{~mm}^{2}$. All patients were intubated transorally for surgery. The tumor was removed with a safety margin of $2 \mathrm{~mm}$, using en bloc or piecemeal techniques depending on the site and size of the tumor and the oropharyngeal exposure. The extent of the tumor was evaluated by visual examination through the operating microscope (Carl Zeiss, Germany), and by palpation with micro-forceps. Intraoperative rigid endoscopy with $0^{\circ}$ and angled telescopes was used when the lesions were difficult to evaluate through the microscope. All patients received $80 \mathrm{mg}$ of methylprednisolone intravenously during the intervention, and $40 \mathrm{mg}$ intravenously of the same drug during the first 2-4 days after surgery.

\subsection{Surgical Margin Assessment}

Examination of frozen sections was conducted when the depth of cancer infiltration was difficult to estimate. After completion of the TLM procedure, the anterior, posterior, and deep margins of the surgical specimen were marked with suture knots and ink, and the specimen was fixed to a soft board and sent to an experienced pathologist. In the case of piecemeal resections, a diagram of the tumor was sent to the pathologist illustrating how the specimen was removed, and a personal review of specimens by the surgeon was conducted with the pathologist if necessary. The pathologist defined the resection margin as negative when the distance of clear pathologic margin was $>$ $0.5 \mathrm{~mm}$. Resection margin $<0.5 \mathrm{~mm}$ was considered as positive, which included cases showing not only apparent tumor involvement of resection margin but also suspicious margins, including suspected zones of tumor involvement in the irregular laser-carbonized zone with thermal artifacts.

\subsection{Postoperative Follow-up}

The patients were followed monthly for the first year, bimonthly for the second year, quarterly for the third year, and biannually for the fourth and fifth years. Flexible laryngoscopy was performed at every postoperative visit and neck CT scans were checked annually. Suspicious lesions identified during the follow-up were checked with micro laryngoscopic examination under general anesthesia.

\subsection{Statistical Methods}

The patient clinicopathologic factors evaluated were age, sex, pathologic $\mathrm{T}$ stage, the extension of tumor to subsites, and the presence of margin invasion. Because our study is on the first-stage assessment, we evaluated only oncologic outcomes for 1-year disease-free survival and organ preservation rate. The prognostic value of the clinicopathologic factors related to the oncologic outcomes was evaluated by univariate analysis. Follow-up ranged from 2 to 18 months (median 8 months). Statistical analysis was performed using PASW version 18 software (SPSS). 


\section{Results}

Of the 30 consecutive patients who underwent TLM, 29 were male and one was female, with an average age of 54.8 (Range 30-79) (Table 1). All patients had an initial endoscopy and either laser or conventional biopsy to confirm the diagnosis. The $\mathrm{T}$ staging for the early oropharyngeal tumors staged 21 patients with $\mathrm{T} 1$ disease and 7 patients with T2 tumors. 4 patients who were detected in the neck dissection, were clinically node positive (N1) on neck CT scan. All patients either drank alcohol or smoked tobacco.

Table 1. Clinical characteristics of the patients

\begin{tabular}{|c|c|c|c|}
\hline \multicolumn{2}{|c|}{ Characteristic } & No. of patients & $\mathbf{N}$ (Total) \\
\hline \multirow[t]{2}{*}{ Sex } & Male & 19 & \multirow[t]{2}{*}{30} \\
\hline & Female & 11 & \\
\hline Age & \multicolumn{3}{|c|}{ Median 54,8 years (Range $30-79$ ) } \\
\hline \multirow[t]{4}{*}{ TMN stage } & T1N0M0 & 21 & \multirow[t]{4}{*}{30} \\
\hline & T2N0M0 & 2 & \\
\hline & T2N0M0 & 3 & \\
\hline & $\mathrm{T} 2 \mathrm{~N} 1 \mathrm{M} 0$ & 4 & \\
\hline
\end{tabular}

The surgical view was adequate for most patients, and those cases with insufficient exposure of the lesion were excluded. A soft diet was given 1 day after the surgery without any significant aspiration events, and nasogastric tube feeding was used for 4 cases T2 N1M0 with neck dissection.

Voice outcomes were assessed with the VHI scale, 24 patients had normal voices, and 6 had mild changes. Only 2 had moderate-to-severe voice changes. There were no aphonic patients. The voice outcome roughly correlates with T staging. Swallowing assessment was predominantly postoperative; however, a few patients with preoperative dysphagia were seen before surgery. First assessment was in nearly all cases 2 days postoperatively. In cases where the patient was unwell the first assessment was up to one week after surgery. Patients whose resection only involved a small part of the tumor recovered normal eating and drinking 1-2 weeks after surgery. Patients with larger resections generally required 1-2 months to reach their potential. Patients with large resections required a longer period of swallowing rehabilitation to learn and review compensation strategies. At their last follow-up, all patients had returned to a normal diet with no discernable difficulty swallowing. There was 2 postoperative bleeding and no significant complications. No other clinical stenosis of the upper airway was found in any of the other patients treated.

The mean hospitalization period was 4.4 days (range 3-8 days). In general, our institutional policy is to discharge a patient the day after surgery, and there were no delayed discharges in this series due to surgical complications. Among the 30 patients whose resection margin was reported by the pathologist, all cases were margin negative, if the intraoperative frozen section had positive margins, surgeon would continue reception until the margins were negative.

During the mean follow-up period of 8 months (range 1-26 months), no patient showed recurrence. The remaining patients are all disease free. The oropharyngeal preservation rate is $100 \%$ for $\mathrm{T} 1 / \mathrm{T} 2$ tumors.

\section{Discussion}

Transoral laser microsurgery can be used in any patient who is fit for general anaesthesia. Post treatment surveillance may be easier following TLM, as the oedema and mucosities associated with External Beam Radiation Therapy (EBRT) are absent ${ }^{9}$. Submucosal disease may be difficult to detect with either modality. TLM and EBRT offer comparable cure rates for early pharyngolaryngeal tumors ${ }^{10,11}$. Comparing the treatment modalities is problematic. TLM allows for accurate staging of the tumor (during resection), whilst radiotherapy relies on the initial micro laryngoscopy for clinical staging. With transoral laser microsurgery, the tumor is resected until the tissue feels and appears healthy under the microscope. In traditional resection methods, the excision line represents the extent of resection, and any histological evidence of tumor at the margins means that the tumor has not been adequately resected. With laser resection, there is a zone of thermal injury to either side of the excision line, and it may, therefore, be difficult for a histopathologist to say with certainty that the tumor is fully excised. It is also difficult in the oropharynx to give the usually accepted millimetres of clearance. Frozen section has in the past been advocated; however, we feel it does not add to the intraoperative management. It may be helpful to send separate specimens from the edge of the resected area which is macroscopically clear of tumor. Transoral laser 
microsurgery, therefore, relies heavily on the surgeon making a competent assessment of the resection.

TLM has certain advantages over "open" surgical procedures. Tracheotomies can have substantial negative impact on postoperative adjustment and quality of life in certain patients following open surgery and are usually not required with TLS ${ }^{12}$. Perioperative morbidity is low and hospitalization is usually short ${ }^{1}$. Deglutition is usually not disturbed, while extended pharyngectomies frequently lead to varying degrees of prolonged aspiration. From the data in the literature that are available to date it is obvious that TLS can be used to treat patients with early-stage oropharyngeal carcinoma successfully.

The organ-preservation rates achieved in our study demonstrate that organ-sparing treatment modalities were frequently successful for patients with local failures after initial TLM. Therefore, our high ultimate local control rates were not achieved at the expense of organ preservation. The quality of voice after laser resection reflects the degree of resection required with more superficial tumors having a better outcome ${ }^{13}$. The literature suggests that voice outcomes in TLM may be either comparable or better compared with EBRT ${ }^{14,15}$. Swallowing outcomes following TLM are good and recovery time relatively short for early oropharyngeal tumors compared to the prolonged discomfort of mucosities and dryness or altered taste following EBRT, which may persist indefinitely. The literature suggests a five-year survival after EBRT to be $85 \%$ for T1-T2 oropharyngeal tumors, and the rate with TLM is comparable ${ }^{5}$. Open pharyngectomy rates are quoted as 4-6\% after open surgery and 9-12\% after radiotherapy. We have only looked at early oropharyngeal tumors so a lower laryngectomy rate would be expected as is the case.

Considering the duration and possible complications of RT and open surgery, TLM may be the preferred treatment modality with shorter hospitalization, minimal morbidity, and comparable local control $(79 \%-92.8 \%)^{1,16}$. Conversely, others demonstrated that extension of tumor to the arytenoids, subglottis, and vocalis muscle had significant impact on local recurrence. Adequate exposure of the lesion in TLM requires experience in handling the suspension laryngoscope. The laryngoscope must be inserted and located at an optimal position using an appropriately sized scope, and repositioning during the procedure may be required in patients with poor exposure or tumors with local extension to the adjacent subsites. The false cord may frequently hinder exposure of the anterior commissure or the ventricle. Laser vestibulectomy should be performed for these cases so that the extent of the tumor and adequate surgical margin may be fully identified. En bloc resection of the tumor may be conducted for small superficial lesions; however, piecemeal resection is useful to evaluate the extent of tumor that may be well identified at the cut surface with different laser carbonizations between SCC and normal mucosa or submucosa. Adequate resection margin in oropharyngeal carcinoma has been considered to be unique (limited as $1-2 \mathrm{~mm}$ ) compared to the general consensus of $>5 \mathrm{~mm}$ to define an adequate margin in SCC of the head and neck ${ }^{3}$. In the absence of a clear definition regarding resection margin of laser pharyngectomy, our pathologist reported a resection margin $<0.5 \mathrm{~mm}$ as positive. The impact of surgical margin on the oncologic outcomes and necessity of postoperative additional treatment or second look procedure after TLM have been debated by many authors with different results and policies ${ }^{17}$.

\section{Conclusion}

Transoral endoscopic $\mathrm{CO}_{2}$ laser microsurgery is a valid technique for treating early oropharyngeal tumors. It has good outcomes for local and distant disease control ${ }^{5,12}$. TLM can be used to treat residual, recurrent, or new tumors even in a previously treated radiotherapy fields. Speech and swallowing results are comparable or better than EBRT.

Laser surgery is associated with time- and costeffectiveness, low morbidity, good local control rates, and excellent retreatment options in cases of local failure. Further prospective studies are still needed to determine the functional outcome for these different treatment options, especially voice, airway and deglutition. However, even when these studies become available with time, there may probably be no "best" therapy for the patient with early oropharyngeal carcinoma, but rather a choice of procedures with different advantages and disadvantages so that the clinician can deal specifically with their patient's individual needs and preferences. 


\section{References}

1. Steiner W. Laser surgery in the ENT field (laser surgery for the treatment of malignant tumors of the upper aerodigestive tract). Archives of oto-rhino-laryngology Supplement $=$ Archiv fur Ohren-, Nasen-und Kehlkopf Heilkunde Supplement. 1986; 2:8-18.

2. Bahannan AA, Zábrodsky $M$, Cerny L, Chovanec M, Lohynska R. Quality of life following endoscopic resection or radio-therapy for early glottic cancer, Saudi Med. J. 2007; 28(4):598-602.

3. Hinni ML, Ferlito A, Brandwein-Gensler MS, Takes RP, Silver CE, Westra WH, et al. Surgical margins in head and neck cancer: A contemporary review. Head and Neck. 2013; 35(9):1362-70. https://doi.org/10.1002/hed.23110. PMid: 22941934.

4. Holsinger FC, McWhorter AJ, Ménard M, Garcia D, Laccourreye O. Transoral lateral oropharyngectomy for squamous cell carcinoma of the tonsillar region: I. Technique, complications, and functional results. Archives of Otolaryngology-Head and Neck Surgery. 2005; 131(7):583-91. https://doi.org/10.1001/archotol.131.7.583. PMid: 16027280.

5. Melong JC, Rigby MH, Bullock M, Hart RD, Trites JR, Taylor SM. Transoral laser microsurgery for the treatment of oropharyngeal cancer: The Dalhousie University experience. J. of Otolaryngology-Head and Neck Surgery. 2015; 44(1):39. https://doi.org/10.1186/s40463-015-00933, https://doi.org/10.1186/s40463-015-0095-1. PMid: 26489752 , PMCid: PMC4618864.

6. Wilkie MD, Upile NS, Lau AS, Williams SP, Sheard J, Helliwell TR, et al. Transoral laser microsurgery for oropharyngeal squamous cell carcinoma: A paradigm shift in therapeutic approach. Head and Neck. 2016; 38(8):1263-70. https://doi. org/10.1002/hed.24432. PMid: 27042800.

7. Motta G, Esposito E, Cassiano B, Motta S. T1-T2-T3 glottic tumors: Fifteen years experience with $\mathrm{CO}_{2}$ laser. Acta Oto-laryngologica. 1997; 117(sup527):155-95. https://doi. org/10.3109/00016489709124062.

8. Rudert H, Werner J. Endoscopic resections of glottic and supraglottic carcinomas with the $\mathrm{CO}_{2}$ laser. European Archives of Oto-rhino-laryngology. 1995; 252(3):146-48. https://doi.org/10.1007/BF00178101. PMid: 7662348.

9. Peretti G, Cappiello J, Nicolai P, Smussi C, Antonelli AR. Endoscopic laser excisional biopsy for selected glottic carcinomas. The Laryngoscope. 1994; 104(10):1276-79. https://doi.org/10.1288/00005537-199410000-00017. PMid: 7934601.
10. Cohen SM, Garrett CG, Dupont WD, Ossoff RH, Courey MS. Voice-related quality of life in T1 glottic cancer: irradiation versus endoscopic excision. Annals of Otology, Rhinology and Laryngology. 2006; 115(8):581-86. https:// doi.org/10.1177/000348940611500803. PMid: 16944656.

11. Weinstein GS, Quon H, O’Malley Jr BW, Kim GG, Cohen MA. Selective neck dissection and deintensified postoperative radiation and chemotherapy for oropharyngeal cancer: A subset analysis of the University of Pennsylvania transoral robotic surgery trial. The Laryngoscope. 2010; 120(9):1749-55. https://doi.org/10.1002/lary.21021. PMid: 20717944.

12. Grant DG, Hinni ML, Salassa JR, Perry WC, Hayden RE, Casler JD. Oropharyngeal cancer: A case for single modality treatment with transoral laser microsurgery. Archives of Otolaryngology-Head and Neck Surgery. 2009; 135(12):1225-30. https://doi.org/10.1001/archoto.2009.185. PMid: 20026820.

13. Thomas L, Jones TM, Tandon S, Carding P, Lowe D, Rogers S. Speech and voice outcomes in oropharyngeal cancer and evaluation of the University of Washington Quality of Life speech domain. Clinical Otolaryngology. 2009; 34(1):34-42. https://doi.org/10.1111/j.17494486.2008.01830.x. PMid: 19260883.

14. Kennedy J, Paddle P, Cook B, Chapman P, Iseli T. Voice outcomes following transoral laser microsurgery for early glottic squamous cell carcinoma, The J. of Laryngology and Otology. 2007; 121(12):1184-88. https://doi.org/10.1017/ S0022215107007554. PMid: 17445355.

15. Haughey BH, Hinni ML, Salassa JR, Hayden RE, Grant DG, Rich JT, et al. Transoral laser microsurgery as primary treatment for advanced-stage oropharyngeal cancer: A United States multicenter study. Head and Neck. 2011; 33(12):1683-94. https://doi.org/10.1002/hed.21669. PMid: 21284056.

16. Canis M, Martin A, Kron M, Konstantinou A, Ihler F, Wolff $\mathrm{HA}$, et al. Results of transoral laser microsurgery in 102 patients with squamous cell carcinoma of the tonsil. Euro. Archives of Oto-rhino-laryngology. 2013;270(8):2299-306. https://doi.org/10.1007/s00405-012-2335-6, https://doi. org/10.1007/s00405-012-2327-6. PMCid: PMC3699705.

17. Ansarin M, Santoro L, Cattaneo A, Massaro MA, Calabrese L, Giugliano G, et al. Laser surgery for early glottic cancer: impact of margin status on local control and organ preservation. Archives of Otolaryngology-Head and Neck Surgery. 2009; 135(4):385-90. https://doi.org/10.1001/ archoto.2009.10. PMid: 19380362. 